\title{
Further Challenges to the "Authentic"/"Hubristic" Model of Pride: Conceptual Clarifications and New Evidence
}

\author{
Colin Holbrook \\ University of California, Los Angeles
}

\author{
Jared R. Piazza \\ University of Pennsylvania
}

\author{
Daniel M. T. Fessler \\ University of California, Los Angeles
}

\begin{abstract}
The Authentic and Hubristic Pride Scales (AHPS) are the methodological core of an influential perspective on pride. The Authentic Pride (AP) scale purportedly measures a distinct facet of pride rooted in attributing success to effort (but not ability), and related to prestige (but not dominance). The Hubristic Pride (HP) scale purportedly measures a complementary facet rooted in attributing success to ability (but not effort), and related to dominance (but not prestige). In the target article, we presented evidence against both profiles. Here, we first examine the counterarguments raised in defense of both the AHPS and the related appraisal-tendency model, then present a new study confirming that AP is elicited by attributions of success to natural ability, and HP is elicited by attributions of success to causes outside the self. It is thus clear that the HP scale measures not pride, but rather evaluations of the self as overclaiming credit or excessively displaying pride.
\end{abstract}

Keywords: pride, arrogance, self-presentation

Tracy, Robins and colleagues pioneered research into the evolved character of pride. Although we criticize both the methods employed in their search for pride's hypothesized dual facets, and the appraisal-tendency model proposed to differentiate the internal structure of these facets, we welcome the proposal that pride may decompose into aspects promoting, respectively, prestige-oriented and dominance-oriented positioning within status hierarchies. However, our data show that the Authentic and Hubristic Pride Scales (AHPS) do not measure a prestige pride rooted in attributing personal success to effort (but not ability) and a dominance pride rooted in attributing personal success to natural ability (but not effort). Tracy and Robins criticize our account as underdeveloped compared with their model of pride. However, it is important not to confuse our critique, which centers on the AHPS instrument, with a proposal about the nature of pride. Rather, our model provides a lens through which to understand the patterns produced by the AHPS.

Our findings reveal serious problems with the AHPS and the related $\mathrm{A} / \mathrm{H}$ appraisal-tendency model. Of gravest concern is the Hubristic Pride (HP) scale, which does not measure pride, but rather perceptions of pride as excessive, either because i) the expression of pride is perceived as unmerited, that is, one is not

Colin Holbrook, Department of Anthropology, University of California, Los Angeles; Jared R. Piazza, Department of Psychology, University of Pennsylvania; Daniel M. T. Fessler, Department of Anthropology, University of California, Los Angeles.

Correspondence concerning this article should be addressed to Colin Holbrook, Center for Behavior, Evolution and Culture, Department of Anthropology, University of California, Los Angeles, 341 Haines Hall, Box 951553, Los Angeles, CA, 90095. E-mail: cholbrook01@ucla.edu genuinely responsible for achievements credited to oneself, or, ii) one realizes that one has displayed pride to others at a proscribed intensity. Note that the former motivation for endorsing HP, highlighted in our "Merited Success versus Unmerited Display" (M/U) model, is antithetical to even the most basic conceptions of pride, for which attributing achievement to oneself is criterial (Tracy \& Robins, 2007). Across several studies (Holbrook, Piazza, \& Fessler, 2014; see also the study below), we document a pattern in which overclaiming credit for success increases HP ratings, and HP ratings are either uncorrelated or negatively correlated with attributing success to internal causes. The second motivation for endorsing $\mathrm{HP}$-acknowledging that pride is excessively displayed-can, but need not, indirectly relate to inner feelings of pride. However, comparing one's conduct to behavioral norms is not equivalent to reporting an emotion: acknowledging acting cocky should not be confused with feeling pride. This "acknowledged cockiness" motive explains Tracy and Robins' (2007, Study 4) finding that "expected feelings" of HP increase following a prime in which one assumes the perspective of a student described as so "naturally talented (i.e., smart)" and confident that they hardly deign to study for an important exam, which they nonetheless ace easily. Such a successful and capable person may indeed be prone to display pride more intensely, but this would not constitute sufficient evidence for a distinct form of pride related to appraising success as owing to one's talent, but not one's hard work.

Regarding the appraisal-tendency model, neither the Authentic Pride (AP) nor HP scales accord with the pattern of appraisal antecedents purported to define them. We found AP to be associated with attributing successes to natural ability as well as to effort, and with rejecting responsibility for failures. Similarly, departing from the predictions of the $\mathrm{A} / \mathrm{H}$ appraisal model, we found that $\mathrm{HP}$ 
is not associated with attributing success to personal ability or causes within the self, but is positively correlated with attributing success to causes outside of the self. In addition, whereas AP is supposed to be distinctly prosocial and prestige oriented, we found AP positively correlated with narcissism and dominance-oriented tendencies. Below, we examine Tracy and Robins' defense of the AHPS, then present a new study addressing what we deem to be their most valid criticism of our prior data.

\section{Regarding the Two-Factor AP/HP Structure}

Tracy and Robins invoke the robustness of the two-factor AP/HP structure as though this were inconsistent with our critique, yet our perspective equally requires two factors. They began their investigations with lay conceptions of the semantic structure of pride as possessing distinct prosocial and antisocial aspects, then empirically confirmed that pride-related words cluster into desirable and undesirable factors. However, it is important to note that cultural schemas concerning emotions often blend subjective experience with their social concomitants. For example, cultural schemas link lust to sin and depravity, social concomitants of, rather than internal facets of, that emotion. Moreover, because the chronic propensity to experience a given emotion is often labeled by cultural schemas, lay conceptions frequently blend subjective experiences and character traits. It is thus unremarkable that Tracy and Robins consistently find two factors for "pride," as the robustness of the HP factor reflects widespread sharing of a cultural schema identifying displays of pride that are excessive relative to norms, along with correspondingly deprecated character traits. ${ }^{1}$ Repeatedly linking pride and arrogance no more sheds light on the internal structure of pride than would repeatedly linking lust and sin illuminate the internal structure of lust.

In an analogous error, Tracy and Robins argue for the validity of the $\mathrm{A} / \mathrm{H}$ model on the basis of its goodness of fit with the traditional distinction between pride as virtue and pride as sin. However, this is separate from the question of whether pride contains distinct prosocial and antisocial facets, as a single emotion can motivate behavior that yields positive and negative social consequences. For example, anger can motivate both prosocial selfsacrifice (e.g., altruistic punishment) and antisocial aggression, but it would be a mistake to conclude that anger is binary on the basis of the presence of cultural schemas that prescribe righteousness and proscribe hotheadedness.

\section{How Does It Feel to Deem One's Pride Excessive?}

Tracy and Robins argue that, because the HP items originated from participants asked to list words related to pride feelings, this scale taps a distinct subjective experience of pride. However, cultural schemas often blend social meaning and emotional experiences. Consequently, when prompted to reflect on feelings of pride, a person may readily imagine behaving in a proscribed, "arrogant" manner. Tracy and Robins (2014) depict us as ascribing no affective motivation to endorsing HP, but instead only a pale "cognitive evaluation of one's arrogant self-presentation" (p. 6). To the contrary, the affective valence associated with this evaluation appears to be predominantly negative, and quite unrelated to the positively valenced emotion pride, insofar as the HP scale is associated with attributing failure (but not success) to oneself, having low self-esteem, fearing negative evaluation, and perceiving oneself as undeserving of merit. Conversely, narcissism also correlated with HP, but not with the aforementioned selfdeprecating pattern (Holbrook et al., 2014, Study 2), and may indeed index experiencing pride. Note, however, that narcissism was also positively associated with AP, and evinced a causal appraisal profile more closely resembling that of AP than of HP.

\section{Authentic Pride, Dominance, and Narcissism}

Contrary to the prosocial portrait of AP presented by the $\mathrm{A} / \mathrm{H}$ model, we found that AP positively correlated with dominance tendencies in contexts of interpersonal conflict (Holbrook et al., 2014, Study 3). Tracy and Robins (2014) question the appropriateness of our employing Sell, Tooby, and Cosmides' (2009) Success in Conflict scale-which was developed to assess the use of coercive strategies to achieve one's ends-on the grounds that "it is better characterized as a measure of generalized social influence or persuasion, combining both dominance and prestige" (p. 14). While the scale does contain some items that appear more closely related to prestige, others appear unambiguously related to dominance. Table 1 presents item-by-item correlations of this scale with AP and HP scores from Study 3 of the target article. Readers can judge for themselves the extent to which these items gauge dominance; AP positively correlated with all of them.

With respect to the correlation between AP and narcissism (Holbrook et al., 2014, Study 2), Tracy and Robins cite two minor methodological concerns and several substantive theoretical distinctions regarding the postulated subtypes of narcissism. The first methodological concern is that we omitted one of the 40 items comprising the Narcissistic Personality Inventory (NPI; Raskin \& Terry, 1988), an error that, though unfortunate, is unlikely to have influenced the composite result. The second concern is that we modified the NPI to be rated linearly rather than as a forced-choice measure. However, our preference for interval-level responses has no obvious bearing on the scale's validity. Space constraints preclude a detailed treatment of the theoretical issues involved in typologizing narcissism, but the crux of Tracy and Robins' position is that AP correlates with healthier forms of narcissism (e.g., related to leadership and self-sufficiency), not unhealthy forms related to feelings of vulnerability, and the composite NPI predominantly indexes these healthier forms. We have no objection to this interpretation, which is consistent with the generally selfenhancing style we found characteristic of AP, and suggestive of pride. However, it bears mentioning that the NPI has been positively correlated with dominance and aggression (e.g., Bushman \& Baumeister, 1998).

Tracy and Robins defend their construal of HP as related to a narcissistically grandiose yet brittle pride concealing a vulnerable

\footnotetext{
${ }^{1}$ All cultures likely include the concept of excessive pride displays, since assertions of superiority are potentially disruptive in any social system. Nonetheless, we can expect that there will be substantial differences between communalist cultures and individualist cultures that prioritize individual aspirations (e.g., Rodriguez Mosquera, Manstead, \& Fischer, 2000). To the extent that communalist cultures emphasize group welfare over individual achievement, these can be expected to have relatively narrow constraints on the expression of pride; to the extent that individualist cultures valorize an aggressive presentation of self, these can be expected to have the loosest constraints on the expression of pride.
} 
Table 1

Correlations of Trait Authentic and Hubristic Pride and the Success in Conflict Scale ${ }^{\dagger}$

\begin{tabular}{lcl}
\hline & Authentic pride & Hubristic pride \\
\hline Success in Conflict Scale & $.30^{* * * *}$ & $.21^{* * * *}$ \\
If I want something, I can usually get it even if others don't want me to have it. & $.38^{* * * *}$ & $.41^{* * * *}$ \\
Other people know not to get in my way. & $.40^{* * * *}$ & $.18^{* * *}$ \\
If another person and I both want something, I will be more likely to get it. & $.26^{* * * * *}$ & $.35^{* * * * *}$ \\
People generally do what I ask them to do. & $.23^{* * * *}$ \\
I don't have much of a problem getting people to do what I want them to do. & $.19^{* * * *}$ \\
I can't get people to do what I want them to do. (Reverse) & $.20^{* * * *}$ \\
When there's a dispute, I usually get my way. & .04 \\
\hline
\end{tabular}

Note. $\quad N=348$.

${ }^{\dagger}$ (Holbrook et al., 2014, Study3). ${ }^{* * *} p<.01 .{ }^{* * * * *} p<.001$.

core of low self-esteem. We maintain that our explanation of the two divergent motives for acknowledging excessive pride provides a more parsimonious and plausible description of why HP simultaneously correlates positively with narcissism and negatively with self-esteem, and refer the reader to the arguments and data presented in the target article. The bottom line is that, whichever interpretation of the link between HP ratings and narcissism one adopts, we have presented convergent evidence that the HP scale does not measure pride.

\section{Sharper Constructs, Not Sharper Brain Scans}

Tracy and Robins argue that, absent brain imaging or similar methods, emotion researchers must use self-report measures, and that such language-based methods suffer the inherent limitation of possibly conflating subjective emotion states with states related to cognitive evaluations of the normative implications of one's behavior. We are more optimistic in this regard. For example, to assess whether the AHPS measures facets of pride, we tested whether AP and HP predict attributing achievements to ability, effort, factors outside the self, and so forth. We also solicited personality measures involving, for instance, public selfconsciousness. Using these straightforward self-report methods, we developed a model of what the AHPS measures; similar methods could be used in the future to vet putative measures of pride (see the target article for further suggestions on assessing/eliciting prestige pride vs. dominance pride). In addition, there is a large literature in psychological anthropology that successfully explores the relationship between emotions and their social valuation using nothing more than interviews. The HP scale consists of the terms arrogant, conceited, egotistical, pompous, smug, snobbish, and stuck-up. We invite readers to discuss with participants whether these terms prototypically refer, on the one hand, to how other people view someone, or, on the other hand, to how people feel when they themselves achieve success.

\section{Experimentally Manipulating Hypothesized Mediators}

Tracy and Robins object that we relied on correlational designs to assess the appraisal tendencies of AP and HP, particularly the causal claim that HP is increased by attributions of achievement to factors external to the self, arguing that experimentally manipulating this proposed mediator would constitute a more compelling test. However, Study $1 \mathrm{~b}$ of the target article did just that. In Study
$1 \mathrm{~b}$, we experimentally manipulated whether a person took credit for a successful outcome that the person either did or did not actually cause. We found that HP increased when the person was not the actual cause and that this increase was mediated by perceptions of the person as undeserving of genuine credit (Holbrook et al., 2014, Study 1b). Tracy and Robins disregard this experimental evidence, and instead propose a new experiment in which HP ratings are compared following manipulations of attributions of success to external causes versus personal talent, as though our perspective does not predict that a person who feels extremely talented would endorse HP. To the contrary, such a design would not adjudicate between Tracy and Robins' position and ours, because our alternative view of the AHPS predicts that, for different reasons, both the "unmerited" and "cocky" pathways should increase HP ratings. The proper test of our prediction would be to compare a neutral condition to experimental conditions in which success is caused by external factors or by superb, effortless talent. Our model predicts that the external cause manipulation should increase HP due to an increase in perceived lack of merit (i.e., via the "unmerited" pathway); the superb talent manipulation should increase HP insofar as it is likely to increase the propensity to overtly display pride (i.e., via the "cocky" pathway). We invite researchers who are unconvinced by the present data to conduct a study along these lines.

\section{State Versus Trait Affect and Appraisal Antedecents}

Tracy and Robins also express concern that our correlational studies focus exclusively on trait rather than state measures of AP and HP (Holbrook et al., 2014, Studies 2 and 3), as event-based appraisal antecedents are inherently related to evocations of state affect. We agree that the lack of state measures presents a significant gap in the data we have presented, and have therefore conducted a new study in which participants recalled an important achievement, reported their state AP and HP, appraised the causes of their achievement, rated the degree to which they felt genuinely deserving of credit for their life success in general, and rated their propensity to outwardly display pride to others.

\section{Study}

A sample of 305 American Mechanical Turk participants (109 female; $M_{\text {age }}=29.33$ ) were asked to recall and write about one of their greatest personal achievements: 
"Please take a few minutes to think about one of the greatest achievements in your life and the reasons for why this achievement came about. Try to remember how it felt to succeed in this important endeavor. Using the box below, please spend a few minutes writing about your achievement, particularly what you think were the causes of your achievement. Make sure to write at least three sentences."

Following this recollection, participants completed the state version of the AHPS, anchored by a 5-point scale (1 = Not at All; $5=$ Extremely; AP $\alpha=.93$; HP $\alpha=.92$ ). Next, participants appraised the causes of their achievement using the same appraisal items as in Study 3 of the target article (effort $\alpha=.57$; ability $\alpha=$ .83; external causes $\alpha=.76$; Holbrook et al., 2014). We then measured participants' endorsement of whether they deserve the level of social status they have experienced in life using Holbrook et al.'s Status Merit Scale ( $\alpha=.78$; e.g., "I'm not especially talented" [reverse-scored]). One item of the scale that had previously referenced earning a place in society "through hard work" was modified to omit this reference to effort. Finally, we measured participants' assessments of their propensity to socially display pride using an original 3-item Pride Display scale $(\alpha=.74$; "When you succeed in life, to what extent do you find yourself walking in a proud way [i.e., swaggering]?"; "When you succeed in life, to what extent do you find yourself wearing a proud facial expression [i.e., smirking]?"; "When you succeed in life, to what extent do you find yourself bringing up your successes in conversation?"). The Pride Display scale was answered according to a 7-point rating scale $(1=$ Not at All; $7=$ Extremely $)$.

\section{Results}

$\mathrm{AP}$ and HP were significantly correlated, $r(305)=.17, p<.01$. We therefore conducted partial correlations of AP and HP to control for shared variance. Part correlations of AP, HP, the appraisal dimensions, and the merit and pride display assessments are presented in Table 2. As we predicted, state AP positively correlated with attributions of success to both personal effort and ability, and negatively correlated with appraisals of external factors as having caused the success. Consonant with our M/U model of the AHPS, state HP was unrelated to appraisals of ability, positively correlated with attributions of success to external causes, and negatively correlated with viewing the self as meriting success. Both state AP and state HP were positively correlated with displaying pride to others following success (see Table 2). As anticipated by our M/U model, state HP was correlated with displaying pride to others despite appraising oneself as not having truly

Table 2

Partial Correlations of State Authentic and Hubristic Pride, Causal Appraisals, Status Merit, and Pride Display

\begin{tabular}{lcc}
\hline & Residual authentic pride & Residual hubristic pride \\
\hline Appraisals & & \\
Effort & $.26^{* * *}$ & $-.16^{* *}$ \\
Ability & $.21^{* * * *}$ & .05 \\
External cause & $-.14^{* *}$ & $.20^{* *}$ \\
Status merit & $.54^{* * *}$ & $-.13^{* *}$ \\
Pride display & $.14^{*}$ & $.21^{* * *}$ \\
\hline
\end{tabular}

Note. $\quad N=305$.

${ }^{*} p<.05 . \quad{ }^{* *} p<.01 .{ }^{* * *} p<.001$. caused a salient success, and as undeserving of merit for success in life. State AP, on the other hand, appears to correlate with pride display for the simple reason that state AP taps feelings of genuine pride in oneself.

Status merit and pride display were positively correlated in this sample, $r(305)=.26, p<.001$. To assess whether the link between AP and pride display was mediated by perceived merit, we ran a follow-up correlation of AP and pride display, partialing out both HP and perceived merit, and found that state AP no longer correlated with pride display, $r(301)=-.01, p>.9$. Thus, pride display appears linked to state AP only to the extent that state AP is associated with perceptions of the self as meriting success. However, when state HP and pride display are correlated, partialing out both AP and perceived merit, HP continues to positively correlate with pride display, $r(301)=.24, p<.001$. This robust relationship appears to reflect the nature of the HP scale as a measure of excessive pride display, rather than a measure of an internal pride state.

\section{Discussion}

Participants rated their state AP and HP following recollection of a life achievement, then appraised the causes of that achievement. The results were consistent with our critique of the AHPS, and inconsistent with Tracy and Robins' A/H model. As in all four studies reported in the target article, AP positively correlated with both ability and effort attributions; as in both prior studies that measured external attributions (Studies $1 \mathrm{~b}$ and 3), HP correlated with attributing achievements to external causes, and, as in all three prior studies that measured perceived merit (Studies 1a, 1b, and 3), HP was negatively associated with meriting credit for successes. Thus, the results of the current study indicate that, at default, the HP scale predominantly measures perceptions of pride display as excessive relative to personal merit, that is, overclaiming. However, this interpretation does not preclude the possibility that, in other contexts, HP might reflect pride display as excessive relative to social norms without impugning merit. Complicating matters still further, participants who appraise themselves as unworthy of merit, and also feel that they have behaved in an intensely "cocky" manner, might conceivably self-apply the HP scale due to overclaiming, cockiness, or both. Indeed, the difficulty in parsing which sort of excessive pride display is being measured is one of the central problems with the HP scale.

\section{Conclusion}

Good theories ought not be shackled to bad methods. Our contention is not that dominance pride and prestige pride do not exist, but rather that the HP scale does not measure pride, and that the AP scale does not measure a form of pride divorced from dominance or from appraisals of the self as naturally gifted.

Looking forward, it is important to note that endorsing the distinction between, and evolutionary significance of, dominance and prestige does not necessitate subscribing to a model of two cleanly differentiated pride adaptations. Instead, as discussed further in the target article, prestige pride may carry the phylogenetic legacy of dominance motivation, such that the derived trait retains some motivational aspects of the ancestral trait, requiring individuals pursuing prestige to actively suppress inclinations toward 
dominance. Precise instruments will need to be developed if we are to adjudicate between such possibilities. Whether phylogenetic inertia has shaped pride to retain dominant propensities or not, it is clear that methodological inertia must not shape future investigation of this emotion.

\section{References}

Bushman, B. J., \& Baumeister, R. F. (1998). Threatened egotism, narcissism, self-esteem, and direct and displaced aggression: Does self-love or self-hate lead to violence? Journal of Personality and Social Psychology, 75, 219-229. doi:10.1037/0022-3514.75.1.219

Holbrook, C., Piazza, J., \& Fessler, D. M. T. (2014). Conceptual and empirical challenges to the "authentic" versus "hubristic" model of pride. Emotion, 14, 17-32.

Raskin, R., \& Terry, H. (1988). A principal-component analysis of narcissistic personality inventory and further evidence of its construct validity. Journal of Personality and Social Psychology, 54, 890-902. doi: 10.1037/0022-3514.54.5.890
Rodriguez Mosquera, P. M., Manstead, A. S. R., \& Fischer, A. H. (2000). The role of honor-related values in the elicitation, experience, and communication of pride, shame, and anger: Spain and the Netherlands compared. Personality and Social Psychology Bulletin, 26, 833-844. doi:10.1177/0146167200269008

Sell, A., Tooby, J., \& Cosmides, L. (2009). Formidability and the logic of human anger. Proceedings of the National Academy of Sciences of the United States of America, 106, 15073-15078. doi:10.1073/pnas .0904312106

Tracy, J. L., \& Robins, R. W. (2007). The psychological structure of pride: A tale of two facets. Journal of Personality and Social Psychology, 92, 506-525. doi:10.1037/0022-3514.92.3.506

Tracy, J. L., \& Robins, R. W. (2014). Conceptual and empirical strengths of the authentic/hubristic model of pride. Emotion, 14, 33-37.

Received July 4, 2013

Revision received September 6, 2013

Accepted September 9, 2013 